\title{
A noncontact measurement technique for the density and thermal expansion coefficient of solid and liquid materials
}

\author{
Sang K. Chung, David B. Thiessen, ${ }^{\text {a) }}$ and Won-Kyu Rhim \\ Jet Propulsion Laboratory, California Institute of Technology, Pasadena, California 91109
}

(Received 18 March 1996; accepted for publication 31 May 1996)

\begin{abstract}
A noncontact measurement technique for the density and the thermal expansion coefficient of refractory materials in their molten as well as solid phases is presented. This technique is based on the video image processing of a levitated sample. Experiments were performed using the high-temperature electrostatic levitator (HTESL) at the Jet Propulsion Laboratory in which 2-3 mm diam samples can be levitated, melted, and radiatively cooled in vacuum. Due to the axisymmetric nature of the molten samples when levitated in the HTESL, a rather simple digital image analysis can be employed to accurately measure the volumetric change as a function of temperature. Density and the thermal expansion coefficient measurements were made on a pure nickel sample to test the accuracy of the technique in the temperature range of $1045-1565{ }^{\circ} \mathrm{C}$. The result for the liquid phase density can be expressed by $\rho=8.848+\left(6.730 \times 10^{-4}\right) \times T\left({ }^{\circ} \mathrm{C}\right) \mathrm{g} / \mathrm{cm}^{3}$ within $0.8 \%$ accuracy, and the corresponding thermal expansion coefficient can be expressed by $\beta=\left(9.419 \times 10^{-5}\right)$ $-\left(7.165 \times 10^{-9}\right) \times T\left({ }^{\circ} \mathrm{C}\right) \mathrm{K}^{-1}$ within $0.2 \%$ accuracy. () 1996 American Institute of Physics. [S0034-6748(96)02609-3]
\end{abstract}

\section{INTRODUCTION}

Density is one of the most fundamental quantities of a material that is intrinsically related to other thermophysical properties. Modern electronic materials industries, for example, are in need of various thermophysical properties of semiconductor melts for the production of higher quality crystals. The density and thermal expansion coefficient affect other thermodynamic values that are needed in the numerical modeling of crystal growth environments. ${ }^{1}$

A number of techniques have traditionally been used to measure the density of high-temperature melts. Among these are the sessile drop, ${ }^{2}$ pycnometric $^{2}$ and dilatometric ${ }^{2}$ archimedian, ${ }^{2,3}$ and maximum bubble pressure ${ }^{2,4}$ methods. Beginning in the 1960s, containerless methods utilizing electromagnetic levitators (EMLs) began to emerge. The main advantage of the containerless method is that it reduces heterogeneous nucleation that is primarily caused by the contacts with container walls, thus, extending the temperature domain of the density measurement to deeply undercooled regions. The EML method, like other noncontact methods, uses imaging techniques to extract the sample volume..$^{5-7}$ However, EML has some difficulties in the accurate determination of sample volume. Some of the drawbacks of the EML for the density measurements are: (i) The stirring effects of the liquid sample caused by the eddy currents makes the sample shape unstable so that the sample volume measurement becomes very inaccurate. Due to this effect one author reported that only $2 \%$ of the collected images turned out to be useful for the volume measurement. ${ }^{6}$ (ii) The test sample is limited to electrical conductors excluding nonconductors and semiconductors. (iii) The closely wound EML coils around the sample make the imaging from the side very difficult. (iv) Finally, the heating and levitation in EML are

${ }^{a)}$ Present address: Physics Department, Washington State University, Pullman, WA 99164. so strongly coupled that it is difficult to reach lower temperature for the density measurements of low melting materials. In contrast, with the high-temperature electrostatic levitator $(\text { HTESL) })^{8}$ that was recently developed at the Jet Propulsion Laboratory (JPL), all of the above difficulties can be resolved. In HTESL the levitated sample is extremely stable $(\sim 10 \mu \mathrm{m}$ fluctuation), and the sample shape is axisymmetric when it is in the liquid form. Due to the axisymmetric nature of the molten sample, only a single image is required to extract the volume information. This makes the volume calculation extremely simple and reliable. The use of modern digital image processing techniques combined with high resolution charged coupled device (CCD) video cameras allows the volume calculations to be done automatically.

In this article we describe for the first time a containerless density measurement technique utilizing the HTESL system combined with digital image analysis. The image analysis techniques used here are similar to those that have been used for pendant drop surface tension measurement. ${ }^{9}$ The technique involves recording a digital image of the drop from a direction perpendicular to the symmetry axis, and fitting the images with spherical harmonic functions to calculate the drop volume. The density is then calculated with the known mass of the sample. The thermal expansion coefficient is calculated using the volume measured as a function of temperature. The underlying definitions for the density, $\rho$ and the thermal expansion coefficient, $\beta$ are

$$
\begin{aligned}
& \rho=m / V, \\
& \beta=\frac{1}{V}\left(\frac{\partial V}{\partial T}\right),
\end{aligned}
$$

where $m$ is the sample mass, $V$ is the sample volume, and $T$ is the sample temperature.

The validity of this density measurement technique utilizing HTESL was demonstrated by performing experiments 


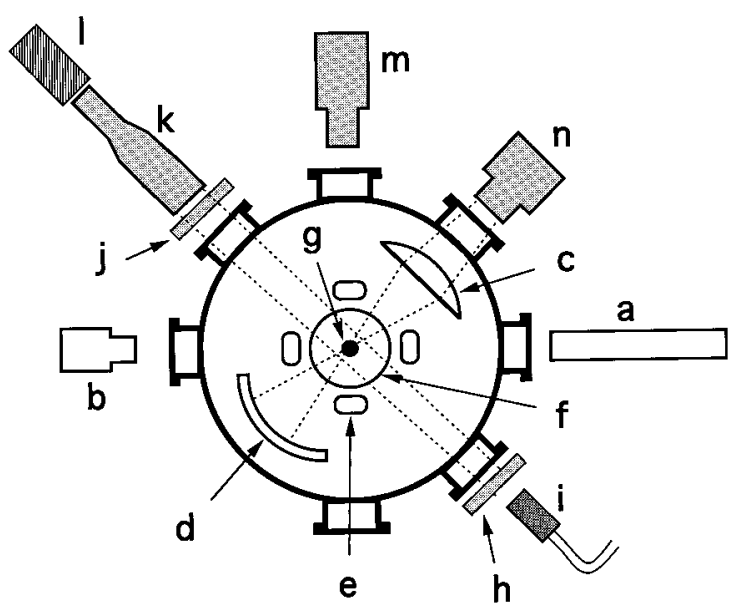

FIG. 1. Schematic diagram of the HTESL system (top view) equipped for the density measurement: (a) HeNe position sensing laser; (b) position sensing detector; (c) focusing lens; (d) focusing reflector; (e) side positioning electrode; (f) top/bottom electrode; (g) sample; (h) backlight diffuser; (i) fiber optical backlight; (j) HeNe blocking filter; (k) long distance microscope; (1) CCD camera; (m) pyrometer; and (n) xenon heating lamp.

on a pure $\mathrm{Ni}$, and they are compared with the results from previous EML studies and other works.

\section{EXPERIMENTAL ARRANGEMENT}

Experiments were performed using the HTESL at JPL. Figure 1 shows a schematic diagram of the HTESL system with an imaging device that is required for the density measurement. The electrode assembly consists of a set of parallel electrodes in which a charged sample, 2-3 $\mathrm{mm}$ in diameter, can be levitated by actively controlling applied electrostatic forces. The electrode assembly is placed at the center of a cylindrical vacuum chamber, which is typically evacuated to $10^{-7}$ Torr.

The sample is heated using a focused $1 \mathrm{~kW}$ xenon arc lamp (PS1000SW-1: ILC Technology) that is capable of raising the sample temperature to about $2300 \mathrm{~K}$. Since the electrostatic levitation is not coupled to the sample heating, the sample can be allowed to cool to room temperature by extinguishing the arc lamp. The cooling phase, therefore, is strictly radiative.

The sample temperature was measured by a custom made single color pyrometer operating at $750 \mathrm{~nm}$ (filter width: $\sim 10 \mathrm{~nm}$ full width at half-maximum) at the $60 \mathrm{~Hz}$ sampling rate. The pyrometer incorporates a photomultiplier tube along with a log ratio amplifier for high sensitivity. A single-reflex viewfinder attached to the pyrometer was equipped with a visual target area that was directed to the point of interest on the surface of the sample. The target spot viewed by the pyrometer was about $1 \mathrm{~mm}^{2}$. The pyrometer was calibrated against a blackbody up to $1000^{\circ} \mathrm{C}$. In order to avoid any interference by the heating lamp, experiments had to be carried out during the cooling period while the lamp was completely blocked or shut off. The emissivity of a sample was determined with respect to the melting temperature, and it was assumed to remain fixed throughout the liquid phase temperature range.

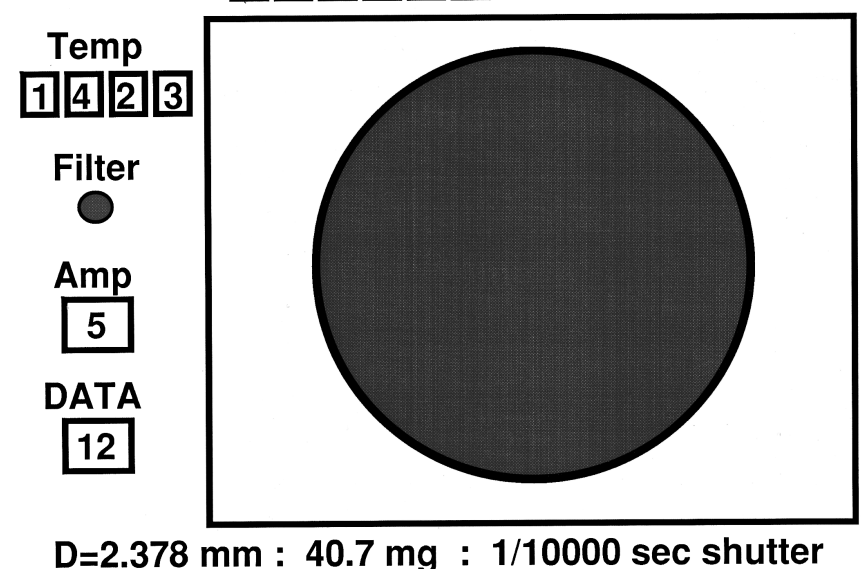

FIG. 2. An example of output image created by overlaying the CCD image with other information such as date, time, and temperature.

The accuracy of the density measurements is critically dependent on the imaging system. The image capture and analysis utilized a high resolution $(570 \times 485)$ black and white CCD video camera (Hitachi Denshi, Ltd.: Model KP-M1) and an eight bit gray scale video frame grabber board (Data Translation: QuickCapture) which plugged into the Nu-Bus slot of the Macintosh computer (Apple: Quadra 950). It has the on-board video memory to store one 640 $\times 480$ element image. In order to magnify the image of a small levitated drop at a minimum working distance of 20 $\mathrm{cm}$, the CCD camera was attached to a long distance microscope (Infinity: Model K2). The microscope allows the levitated sample image to fill the area of the CCD chip. A collimated fiber optical light source (Fiberoptics Specialties, Inc.: $250 \mathrm{~W}$ ) was used to back light the levitated sample in order to obtain high-contrast images even at the temperature where the sample glows brightly. In order to capture sharp images, the shutter speed of the camera was maintained at $10^{-4} \mathrm{~s}$. To block the scattered light from the position feedback laser that reflected off the sample surface, a HeNe line blocking filter was inserted in the optical path between the sample and the microscope. A separate Macintosh II computer equipped with a video overlay card (Computer Friends: TV Producer) is used to superimpose the time and temperature information on the CCD image. Figure 2 shows a typical output of an overlaid video frame that was used for the density measurement. A video time base corrector (SONY: MPU-F100) was used to stabilize the paused frame transfer from VCR to computer.

\section{IMAGE ANALYSIS}

In order to derive accurate volume information from a digital image it is necessary to incorporate a consistent and fail proof algorithm. The first step in the image analysis process is to locate the edge of the drop. A set of 400 equally spaced radial vectors are created centered on the approximate drop center $\left(x_{0}, y_{0}\right)$, 


$$
\begin{gathered}
\left.\begin{array}{c}
x_{i j}=r_{i} \sin \theta_{j}+x_{0} \\
y_{i j}=r_{i} \cos \theta_{j}+y_{0} \\
\theta_{j}=2 \pi j / 400
\end{array}\right\}, \quad j=1, \ldots, 400, \\
r_{i}=i \delta r, \quad \delta r=1,
\end{gathered}
$$

where $i$ is the index of the pixel along the radial direction, and $x, y$ are the horizontal and vertical coordinates, respectively. The intensity at spacings of one pixel along each vector is determined using bilinear interpolation of the adjacent pixel intensities. The drop edge is detected by searching for the maximum intensity gradient along each vector to yield a set of 400 edge points, $\left(x_{j}, y_{j}\right)$. The averages of the $x$ and $y$ coordinates of the edge points are taken as a new estimate of the drop center. New radial vectors are set up centered on this new estimate, $x_{0}$ and $y_{0}$, and edge detection is repeated once more.

The edge points are fit with the spherical harmonic functions through sixth order as

$$
R(\theta)=\sum_{n=0}^{6} c_{n} P_{n}^{0}(\cos \theta),
$$

where $P_{n}^{0}(\cos \theta)$ are the $n$th order Legendre polynomials, and $c_{n}$ are the coefficients that must be determined by the fitting procedure. The experimental edge coordinates are converted to polar coordinates,

$$
\begin{aligned}
& R_{j}=\left[\left(x_{j}-X_{0}\right)^{2} L_{x}^{2}+\left(y_{j}-Y_{0}\right)^{2} L_{y}^{2}\right]^{1 / 2}, \\
& \theta_{j}=\sin ^{-1}\left[\frac{\left(x_{j}-X_{0}\right) L_{x}}{R_{j}}\right],
\end{aligned}
$$

where $L_{x}$ and $L_{y}$ are the horizontal and vertical magnification factors $(\mathrm{cm} /$ pixel $)$ determined from the calibration procedure. The following objective function is minimized to determine the coefficients of the Legendre polynomials,

$$
F\left(X_{0}, Y_{0}, c_{0}, \ldots, c_{6}\right)=\sum_{j=1}^{400}\left[\frac{R_{j}-R\left(\theta_{j}\right)}{\sigma_{j}}\right]^{2},
$$

where $X_{0}$ and $Y_{0}$ are the pixel coordinates of the origin of the polar coordinate system, and $\sigma_{j}$ is the measurement error of the $j$ th data point. The values of $X_{0}$ and $Y_{0}$ are initialized to the average $x$ and $y$ coordinates of the experimental edge points. The objective function $F$ is minimized with respect to $X_{0}$ and the coefficients $c_{0}$ through $c_{6}$. The fitting function $R(\theta)$ given by Eq. (4) is linear in the coefficients, thus, allowing for a linear least-squares method to determine the optimal values of the coefficients for a given value of $X_{0}$. A one-dimensional minimization of $F$ with respect to $X_{0}$ is performed using a parabolic interpolation algorithm. Each time $X_{0}$ is changed, the polar coordinates of the experimental edge points are recomputed according to Eq. (5) and $F$ is minimized with respect to the Legendre polynomial coefficients by linear least squares. The parameter $Y_{0}$ does not need to be optimized since the fitting function can accommodate a vertical shift in the coordinate system.

Following optimization of the coefficients of the Legendre polynomials, the volume is computed by integrating the optimal profile as follows:

$$
V=\frac{2 \pi}{3} \int_{0}^{\pi} R(\theta)^{3} \sin \theta d \theta .
$$

A rough estimate of the error in the volume calculation arising from random errors in the position of the edge points can be obtained by considering the uncertainty in $R(\theta)$. For a spherical sample, an uncertainty in $R$ of $\delta R$ leads to an uncertainty in volume of $4 \pi R^{2} \delta R$. The relative error in the volume is then

$$
\frac{\delta V}{V}=\frac{4 \pi R^{2} \delta R}{4 \pi R^{3} / 3}=\frac{3 \delta R}{R} .
$$

A typical image of a molten Ni sample gives $R=160$ pixels. If $\delta R$ is taken to be one pixel, then the percent uncertainty in the volume is $1.9 \%$. The actual uncertainty in $R$ may be more or less than one pixel depending on image quality. This error estimate applies only to random errors in edge points arising for example from digital noise in pixel intensities.

\section{EXPERIMENTAL PROCEDURE}

Before the experiment, the imaging system is aligned with respect to the HTESL, which in turn is aligned with the gravity vector. The first step was to align the axis of the parallel levitation electrodes with respect to the gravity vector. A horizontal level sensor was used for this task. The second step was to rotationally align the CCD camera such that an image of either electrode produces an edge that is aligned with a row of pixels. A computer generated horizontal line that was overlaid with the flat electrode image was used to aid this process. These procedures ensure that the symmetry axis of the levitated molten sample is aligned with the vertical pixels of the CCD camera as well as with the gravity vector.

A roughly spherical nickel sample (Electronic Space Products Int.: $99.99 \%$ purity) was inserted into the levitation chamber along with a calibration ball (BallTech: the calibration ball size was specified to within $\pm 2.5 \mu \mathrm{m})$ and the chamber was evacuated to the low $10^{-7}$ Torr. The Ni sample preparation method and the levitation melting in the HTESL was described in detail previously. ${ }^{10}$ The magnification of the microscope, the brightness, contrast, and focus of the image were then adjusted. Once the optimum settings were achieved, all adjustments to the imaging system were fixed until the experiment was finished. Images of the levitated calibration sphere were recorded onto video tape for later processing.

After the calibration sphere was retrieved, the Ni sample was levitated and heated by the arc lamp until the sample was fully molten. The sample was then allowed to cool in a purely radiative manner by extinguishing the arc lamp. A typical cooling curve measured for $\mathrm{Ni}$ is shown in Fig. 3. The collected raw temperature data is then adjusted by changing the emissivity value until the highest value in the isothermal region, immediately following the recalescence (indicated as No. 4 in Fig. 3), matched the melting temperature $\left(T_{m}=1453{ }^{\circ} \mathrm{C}\right)$ of $\mathrm{Ni}$. The temperature reading in the isothermal postrecalescence region did not remain constant. It fell about $7 \mathrm{~K}$ over $1.8 \mathrm{~s}$ duration, which is $0.48 \%$ of $T_{m}$. 


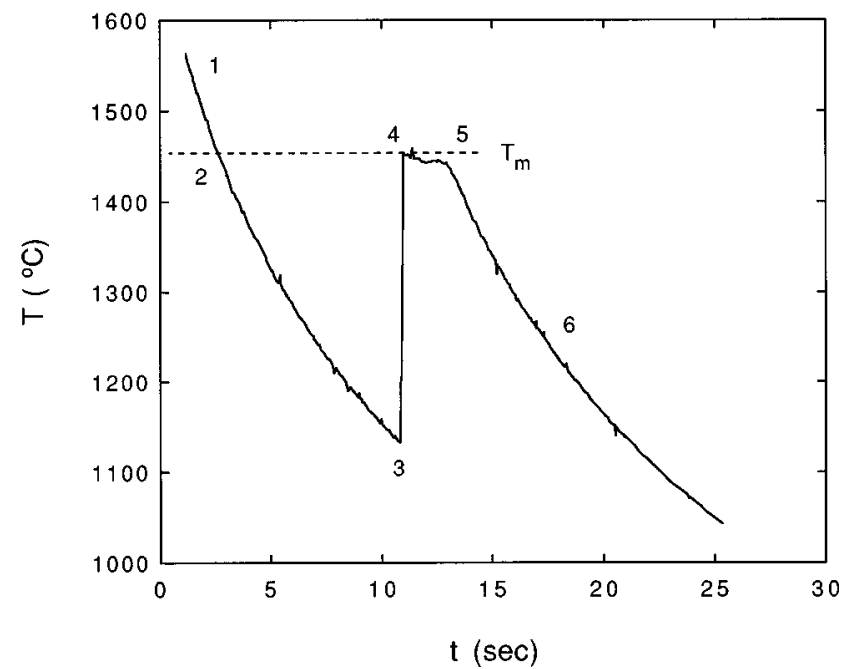

FIG. 3. A radiative cooling curve observed for the nickel sample used in this experiment: (1-2) superheated stage, (2-3) undercooled stage, (3-4) recalescence stage, (4-5) solidification stage, and (5-6) further cooling after solidification.

This may be due to the changing spectral emissivity during the solidification process. The emissivity determined for the $\mathrm{Ni}$ sample at $750 \mathrm{~nm}$ spectral band was 0.449 . This emissivity value was then used in subsequent experiments for the live temperature readings from the pyrometer. The experiments consisted of heating the Ni sample to a desired temperature above $T_{m}$, then extinguishing the arc lamp that enabled the simultaneous acquisition of temperature and the sample images by recording them through VCR in a format described earlier (Fig. 2). Following the recording of the images, the sample was cooled and retrieved. After the chamber was opened the sample was weighed once again for possible mass loss through evaporation. In each cycle of heating and cooling, there was about $0.6 \%$ loss of mass through evaporation.

After the experiment, video images of the calibration sphere and the Ni sample were transferred to the hard disk of the computer using the video frame grabber. Several digital images of the calibration sphere were used to average each of the vertical and the horizontal magnification factors for the optical system.

The experiment involved ten superheating and cooling cycles for the Ni sample. Image data were analyzed only for the eighth cycle since it attained the deepest undercooling prior to solidification. A total of 281 sequential images of the $\mathrm{Ni}$ sample were captured from video tape and analyzed for this cycle. This number represents a subset of the total number of frames available on the video tape since some frames could not be used because of bad temperature or time information (sometimes, a frame was captured during the update procedure of the temperature or the time). An image analysis software based on the algorithm described in the previous section was launched to automatically analyze the volume for each image collected, with the calculation taking about 20 s per image. The software automatically created a text file containing the time, the temperature, and the volume information for each image. The density and the thermal expan-

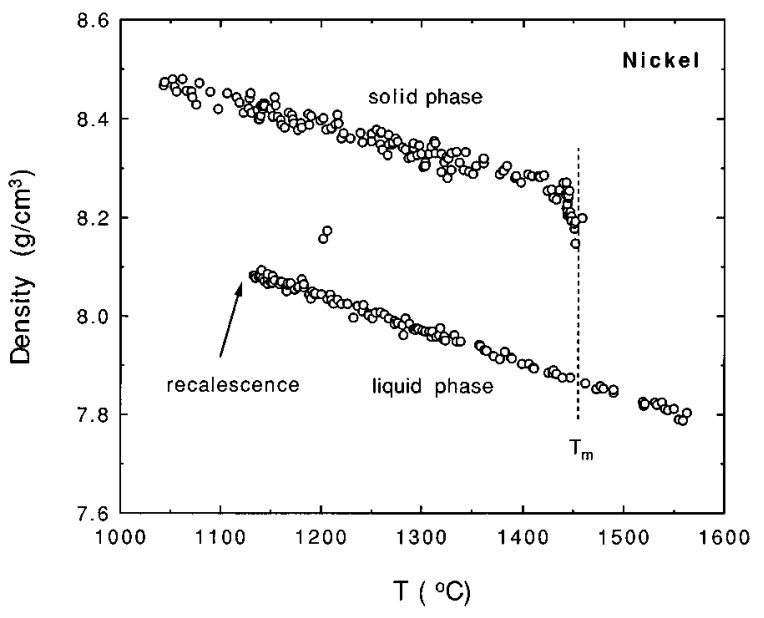

FIG. 4. Density of the nickel sample analyzed for the cooling period.

sion coefficient for the $\mathrm{Ni}$ sample were then obtained by using Eqs. (1) and (2). The mass of the Ni sample measured before and after the experiment that involved ten superheating and cooling cycles were 42.5 and $40.2 \mathrm{mg}$, respectively. It is, therefore, assumed that each cycle is responsible for a mass loss of $0.23 \mathrm{mg}$ due to evaporation. The mass of the $\mathrm{Ni}$ sample used to calculate the density was taken as the initial mass, subtracted by the mass loss expected through the eight cycles, giving $40.66 \pm 0.23 \mathrm{mg}$.

\section{EXPERIMENTAL RESULTS}

Plotted in Fig. 4 is the density of the Ni sample measured during the cooling phase. Notice the isothermal change in density following recalescence during which a mixture of solid and liquid coexist. The solidification process generally deforms the shape of the drop somewhat so that the sample is no longer perfectly axisymmetric. The accuracy of the volume measurement for the solidified sample is, therefore, expected to be reduced relative to that of the molten sample. This effect is seen in Fig. 4 as an increased scatter in the data following solidification. Our result for the liquid density of $\mathrm{Ni}$ at the melting point $\left(7.87 \pm 0.06 \mathrm{~g} / \mathrm{cm}^{3}\right)$ is in good agreement with the handbook value ${ }^{11}\left(\sim 7.84 \mathrm{~g} / \mathrm{cm}^{3}\right)$. When the density data for the solid phase of $\mathrm{Ni}$ are linearly extrapolated to room temperature $\left(25^{\circ} \mathrm{C}\right)$, they yield a value of 9.04 $\mathrm{g} / \mathrm{cm}^{3}$, which is somewhat higher than the handbook value ${ }^{11}$ of $8.902 \mathrm{~g} / \mathrm{cm}^{3}$.

Figure 5 shows the density of the molten $\mathrm{Ni}$ from the present work compared with earlier studies that used EML and other methods. ${ }^{3,4,6}$ The $\partial \rho / \partial T$ measured here is somewhat less than other results. One can think of several potential causes for such a discrepancy. One of them might be the presence of radial temperature gradients within the sample that will occur during fast, radiative cooling. Such gradients would cause the measured density to be lower than it should be for a given surface temperature since the interior is hotter than the surface. A conservative estimate of the temperature gradient in this system indicates a maximum temperature difference between the surface and interior of less than $10 \mathrm{~K}$. In order to observe the temperature gradient effect over the density vs temperature curve, a simple experiment was de- 


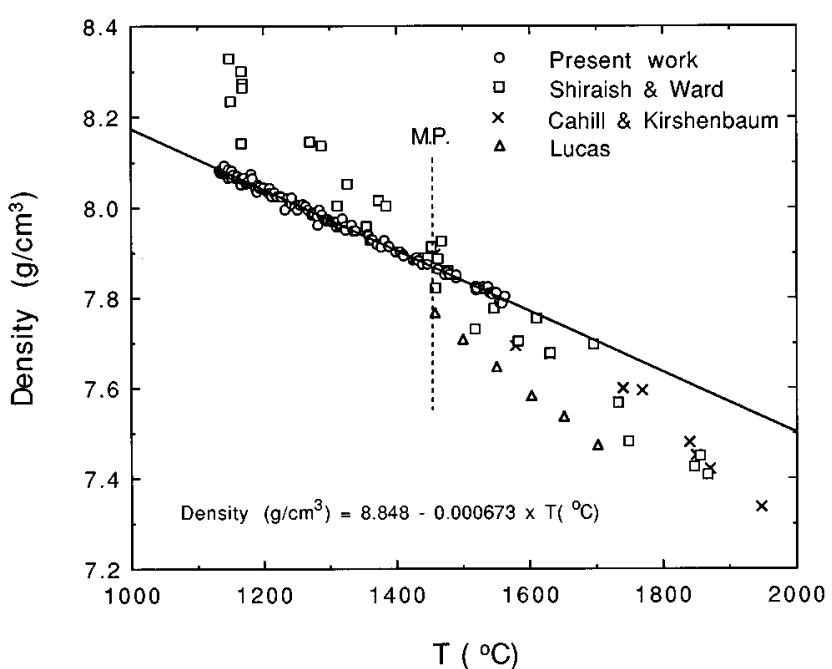

FIG. 5. Density of the molten Ni from the present work compared with earlier studies that used EML (Shiraish), maximum bubble pressure (Lucas), and archimedian (Cahill and Kirshenbaum) methods.

vised. The experiment consisted of performing a series of superheating and radiative cooling processes on the same sample in which the only difference was that each cooling cycle is preceded by different equilibrium temperature. The idea was to construct a quasistatic volume expansion curve as a function of temperature by integrating the volume data taken only at initial equilibrium temperature for each cycle, thereby, effectively eliminating the surface to the bulk (radial) temperature gradient that may exist in the case of rapid cooling. A Ni-Zr alloy $\left(T_{m}=960{ }^{\circ} \mathrm{C}: 24 \% \mathrm{Ni}\right.$ and $76 \% \mathrm{Zr}$ by composition) was chosen for a test sample because of its low vapor pressure around its melting temperature. For the duration of 18 superheating and undercooling cycles with temperatures ranging from 780 to $1390{ }^{\circ} \mathrm{C}$, it lost less than $0.02 \%$ of the initial mass. Figure 6 shows the inverse of the volume expansions (normalized with the volume at $780{ }^{\circ} \mathrm{C}$ for convenience) made by radiative cooling and by quasistatic cooling, both measured with the same $\mathrm{Ni}-\mathrm{Zr}$ sample.

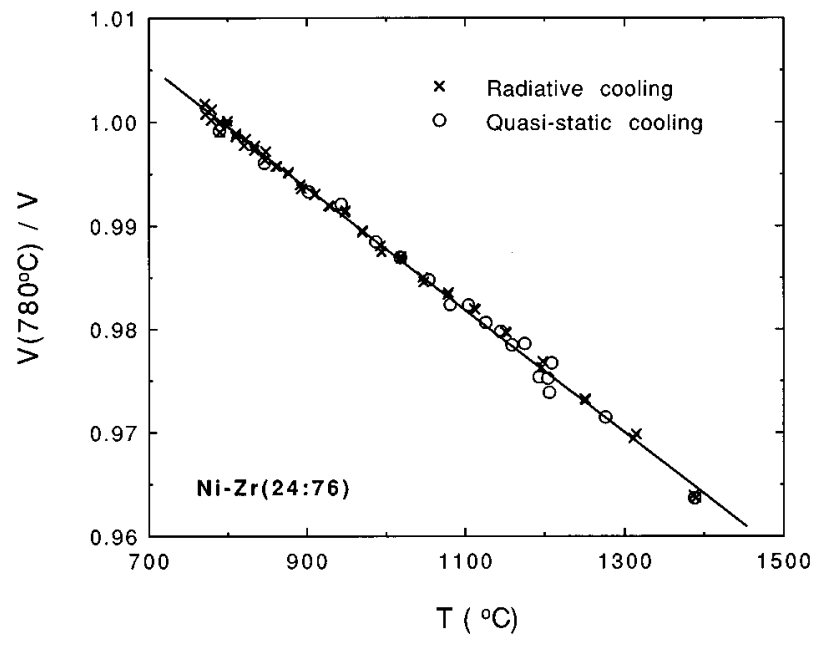

FIG. 6. Inverse of the volume expansions (normalized with the volume at $780^{\circ} \mathrm{C}$ for convenience) made from quasistatic cooling and the purely radiative cooling are compared for a $\mathrm{Ni}-\mathrm{Zr}$ alloy sample.

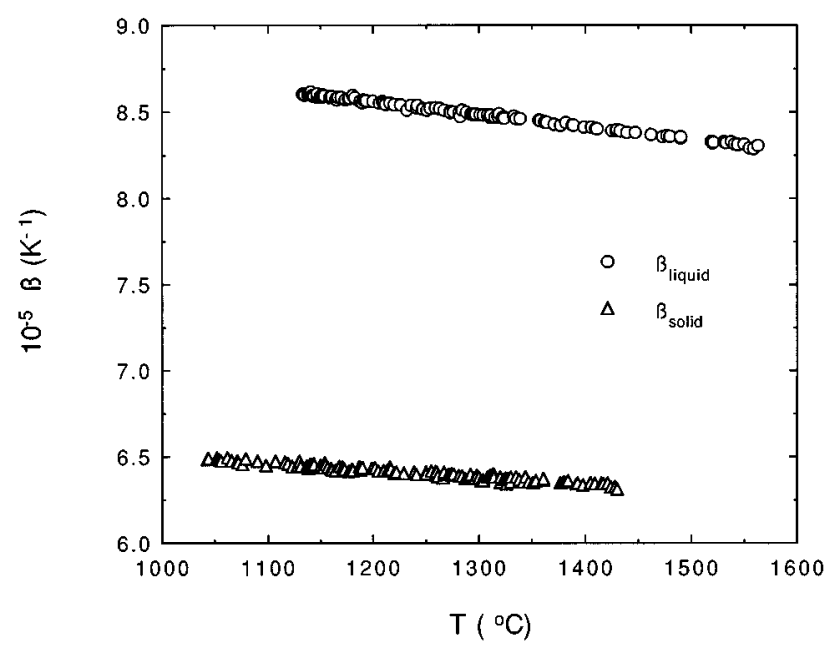

FIG. 7. Volumetric thermal expansion coefficient of Ni.

The overlapping results from two different cooling methods indicate that there was no significant radial temperature gradient effect for the radiatively cooled $\mathrm{Ni}-\mathrm{Zr}$ sample. Due to the fact that the $\mathrm{Ni}-\mathrm{Zr}$ alloy has the lower thermal conductivity than the pure $\mathrm{Ni}$ ( $\mathrm{Zr}$ is four times less thermally conductive than $\mathrm{Ni}$ ), one can safely assume that the $\mathrm{Ni}$ of similar size would also have negligible radial temperature gradient when cooling radiatively. The radial temperature gradient effect within the Ni, thus, does not explain the observed difference of $\partial \rho / \partial T$ of present data and that of earlier works.

Figure 7 shows the volumetric thermal expansion coefficient for $\mathrm{Ni}$ in the liquid and solid states derived from the volume and temperature measurements. Since the mass of the sample does not enter in Eq. (2), rather accurate values are obtained for the thermal expansion coefficients. The density and thermal expansion coefficient for liquid and solid $\mathrm{Ni}$ as a function of temperature can be expressed as follows:

$$
\begin{aligned}
& \rho_{l}=8.848+\left(6.730 \times 10^{-4}\right) \times T \pm 0.8 \% \\
&\left(1130<T<1565^{\circ} \mathrm{C}\right), \\
& \beta_{l}=\left(9.419 \times 10^{-5}\right)-\left(7.165 \times 10^{-9}\right) \times T \pm 0.2 \% \\
&\left(1130<T<1565^{\circ} \mathrm{C}\right), \\
& \rho_{s}= 9.031+\left(5.361 \times 10^{-4}\right) \times T \pm 1.0 \% \\
&\left(1045<T<1455^{\circ} \mathrm{C}\right), \\
& \beta_{s}=\left(6.917 \times 10^{-5}\right)-\left(4.107 \times 10^{-9}\right) \times T \pm 0.4 \% \\
&\left(1045<T<1455^{\circ} \mathrm{C}\right),
\end{aligned}
$$

where the units are in $\mathrm{g} / \mathrm{cm}^{3}$ for $\rho, \mathrm{K}^{-1}$ for $\beta$, and ${ }^{\circ} \mathrm{C}$ for $T$.

\section{DISCUSSION}

In determining the accuracy of the present density measurement technique, three factors were considered: first, the error due to the scattering of the measured volume of the calibration sphere plus some residual optical error; second, the random noise and the systematic error of the pyrometer that relate to the density measurement error; and third, the 


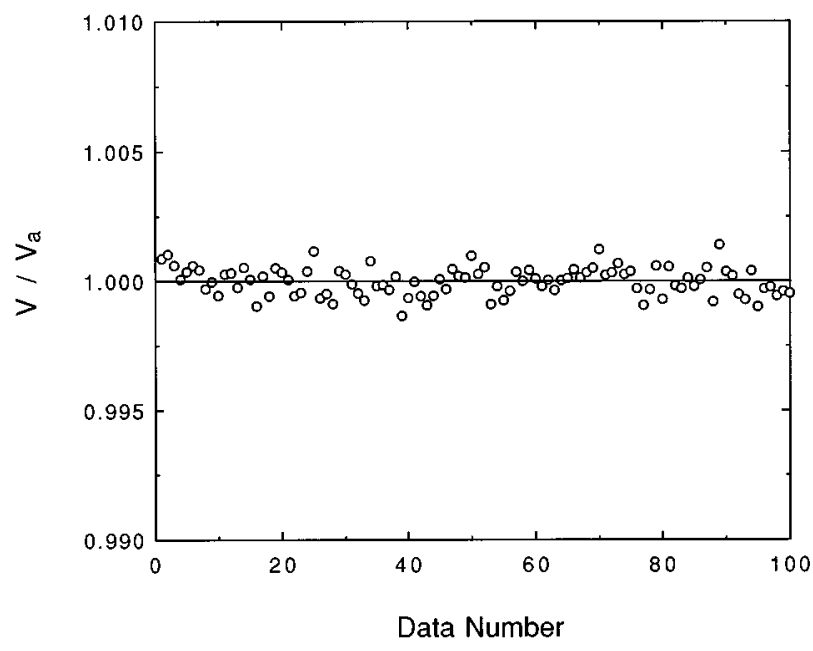

FIG. 8. Scattering of the volumes made from 100 calibration images measured separately. Volumes are divided by the average value.

error caused by the measurement of the mass of the sample. The accuracy of $\beta$, since it does not involve the mass of the sample, depends only on the accuracy of the volume measurement and the pyrometer.

In order to test the accuracy of the present technique, 100 images of a calibration sphere at room temperature were captured from video tape and analyzed for its volume. The scattering of the analyzed volume is shown in Fig. 8 in the form of dimensionless quantity, the volume divided by the average value. The result showed the volume within $0.06 \%$ standard deviation. The dispersion in these measurements arises from the collective noise in the imaging system that is generated by fluctuations in lighting, nonuniformity in focusing, optical distortion, camera misalignment, and VCR and frame-grabber electronics.

In the case of a solidified drop, additional noise can arise if the drop shape is nonaxisymmetric due to surface irregularities caused by the surface depression or nonuniform crystalline structure combined with the sample rotation. In this case, the scattering is calculated directly from the deviation from the best fit curve. A sample glowing at high temperature is another source of error in determining the edge coordinates. Even though the edge detection relies on the intensity gradient rather than the absolute intensity of the digital image, a simulated sample glowing tests indicated as much as $0.05 \%$ discrepancy of volume. The temperature gradients along the surface arising from nonuniform heating are estimated to be negligible for the type and size of samples used here. The sample glow effect is also kept minimal in this work $(<0.02 \%)$ by incorporating the intense background light and the fast shutter $(1 / 10000 \mathrm{~s})$ speed of the CCD camera. The combined accuracy of the volume measurement technique for the liquid sample then reaches about $0.1 \%$.

Accurate temperature measurement is another crucial factor when measuring the temperature dependence of the density. The maximum random noise in the pyrometer within a single frame time was approximately $\pm 5 \mathrm{~K}$ except at the point of rapid recalescence. Apart from the random noise there was a systematic error caused by the temperature cali- bration. The uncertainty caused by the temperature calibration was approximately $7 \mathrm{~K}$ arising from the variation of the temperature in the isothermal postrecalescence region. This error incorporates the spectral emissivity difference between the liquid and the solid states of the sample. The relative error in the density that arises from an error in the temperature reading can be expressed as

$$
\frac{\delta \rho}{\rho}=\frac{1}{\rho}\left(\frac{\partial \rho}{\partial T}\right) \delta T=-\beta \delta T .
$$

A maximum temperature error $(\sim \pm 12 \mathrm{~K})$ for molten $\mathrm{Ni}$ then corresponds to a density error of approximately $0.1 \%$.

Accuracy of the sample mass depends on the vapor pressure of the molten sample at the operating temperature. Therefore, the largest error in the density measurement from this experiment is expected to arise from the uncertainty in mass that is estimated to be $\pm 0.57 \%$, the mass loss associated with a single superheating and cooling cycle. This is an error for a given sample that can be arbitrarily reduced if one can improve the method of measurement. This error is expected to be insignificant for materials with low vapor pressure and can be significantly reduced for high vapor pressure materials by conducting the experiment with fewer cycles of superheating and cooling.

The accuracy of density measurements for solidified samples also depends on the type of material being processed. Materials that solidify by a first order phase transition, such as $\mathrm{Ni}$, can show surface roughening arising from interdendritic shrinkage. However, due to its maintenance of roughly the same axisymmetrical shape upon solidification, density measurement is still possible with some loss of the accuracy. With material such as silicon whose volume expanded as it solidified, it not only lost the axisymmetricity but it underwent a rather dramatic shape distortion. In this case, the present density measurement technique was useful only for the liquid phase of the sample. Glass forming materials on the other hand, solidify by a continuous hardening process and are, thus, expected to show less deformation. The glass forming metal alloy, $\mathrm{Zr}_{41.2} \mathrm{Ti}_{13.8} \mathrm{Cu}_{12.5} \mathrm{Ni}_{10.0} \mathrm{Be}_{22.5}$ (Ref. 12) $\left(\mathrm{Tm}=720{ }^{\circ} \mathrm{C}\right)$, was seen to preserve its axisymmetric shape as well as the surface smoothness during solidification. In this case, high accuracy in the density measurements was maintained all the way to room temperature. The density result on molten silicon and the density results on glass forming alloys will be published in near future. In summary, the highest accuracy that can be achieved for the density measurement using the current system will be the case for nonevaporative material in its liquid state. The accuracy in this case will be better than $0.2 \%$.

It should be emphasized that the current system can be modified in several ways to improve the accuracy. One obvious improvement may be achieved by reducing the collective noise generated by the optical system. Additional improvements may include the reduction of floor vibration, the use of a more spatially uniform background light source, and the use of a higher quality video recording/retrieving system. It is expected that such improvements can reduce the error in the density measurement to less than $0.15 \%$. An even higher level of accuracy could be achieved by increasing image 
resolution by replacing the current $C C D$ camera with a film camera (35 $\mathrm{mm}$ or high speed motion picture) or by a higher resolution CCD camera $(>1000 \times 1000$ pixels $)$. Film images can be digitized with a high resolution film scanner to provide at least four times the resolution of the current CCD camera. The accuracy in the pyrometer reading will be the largest source of error if all of the above improvements are accomplished.

One significant difference between the density measurement approach described here and in previous studies is that all the data points for the volume measurement were taken from a single sample. So far we have determined the densities of nickel, silicon, ${ }^{13}$ aluminum, titanium alloy (Ti64), nickel-zirconium alloy, and $\mathrm{Zr}_{41.2} \mathrm{Ti}_{13.8} \mathrm{Cu}_{12.5} \mathrm{Ni}_{10.0} \mathrm{Be}_{22.5}$ glass forming alloy. Some of these results will be published elsewhere.

\section{ACKNOWLEDGMENTS}

The authors thank Mr. Daniel Barber for his technical support throughout the experiments including the sample preparations and Dr. Erik Spjut for his earlier construction of the pyrometer. The authors acknowledge Dr. Yong J. Kim for his assistance in the data taking part of the experimental work. The authors also especially thank Dr. Kenichi Ohsaka for his arduous checking on the reliability of the volume analysis algorithm. This work was carried out at the Jet Propulsion Laboratory, California Institute of Technology, under contract with the National Aeronautics and Space Administration. Reference herein to any specific commercial product, process, or service by trade name, trademark, manufacturer, does not constitute or imply its endorsement by the United States Government or the Jet Propulsion Laboratory, California Institute of Technology.

${ }^{1}$ S. Nakamura and T. Hibiya, Int. J. Thermophys. 13, 1061 (1992).

${ }^{2}$ T. Iida and R. I. L. Guthrie, The Physical Properties of Liquid Metals (Oxford University Press, New York, 1988).

${ }^{3}$ A. D. Kirshenbaum and J. A. Cahill, Trans. Am. Soc. Metall. 56, 281 (1963).

${ }^{4}$ L. D. Lucas, Doctoral thesis, University of Paris (1962).

${ }^{5}$ A. E. El-Mehairy and R. G. Ward, Trans. Metall. Soc. AIME 227, 1226 (1963).

${ }^{6}$ S. Y. Shiraishi and R. G. Ward, Can. Metall. Q. 3, 117 (1964).

${ }^{7}$ T. Saito and Y. Sakuma, Trans. Iron Steel Inst. Jpn. 9, 118 (1969).

${ }^{8}$ W. K. Rhim, S. K. Chung, D. Barber, K. F. Man, G. Gutt, A. Rulison, and R. E. Spjut, Rev. Sci. Instrum. 64, 2961 (1993).

${ }^{9}$ D. B. Thiessen and K. F. Man, Int. J. Thermophys. 16, 245 (1995).

${ }^{10}$ A. Rulison and W. K. Rhim, Rev. Sci. Instrum. 65, 695 (1994).

${ }^{11}$ R. C. Weast and M. J. Astle, CRC Handbook of Chemistry and Physics, 62nd ed. (Chemical Rubber, Boca Rotan, FL, 1981).

${ }^{12}$ A. Peker and W. L. Johnson, Appl. Phys. Lett. 63, 2342 (1993).

${ }^{13}$ W. K. Rhim, S. K. Chung, A. J. Rulison, and R. E. Spjut, Int. J. Thermophys. (to be published). 